\title{
Analysis of Demand Changes in a University Counseling Center Telehealth System
}

\author{
Aleksandra Orlovic, Michelle Alvarado, Sara Nash, Alvin Lawrence, \& \\ Ernesto Escoto \\ University of Florida
}

Faculty Mentor: Michelle Alvarado, Department of Industrial and Systems Engineering

\begin{abstract}
The Counseling and Wellness Center (CWC) offers various types of mental health appointments for students at the University of Florida. The CWC is implementing a new walk-in system for student appointments to increase the timeliness and accessibility of first appointments. Due to the COVID-19 pandemic, the CWC shifted to offer telehealth appointments, primarily through Zoom. The research objective is to conduct a data analysis of historical appointment data before the shift to telehealth and after the shift to telehealth to understand how appointment demand changed during the pandemic. The data analysis breaks down the data by appointment type, weekday, and time of day. This project collaborates with staff at the CWC and has the goal of helping the CWC better understand demand patterns, so they can better anticipate appointment demand and serve the UF student population in a timely manner.
\end{abstract}

Keywords: demand data analysis, telehealth, student counseling

\section{Analysis of Demand Changes in a University Counseling Center Telehealth System}

The Counseling and Wellness Center (CWC) is one of the main resources for general counseling and mental health at the University of Florida, especially for students. Prior to the COVID-19 pandemic, the CWC offered the majority of their appointments as in-person appointments. As a result of the pandemic, the CWC switched to primarily offer their appointments as telehealth appointments, primarily through Zoom. The U.S. Department of Health and Human Services (HHS) defines telehealth as "the use of electronic information and telecommunication technologies to provide care when you and the doctor are not in the same place as the same time" (United States Department of Health and Human Services, 2021, p.1).

In the past, the CWC has struggled to meet appointment demand, as often the demand for appointments at the CWC was greater than the available counselors and resources. Previously, 
students who would like a walk-in non-emergency appointment at the CWC would have to go to one of the CWC locations on campus, sign-in and hope to be able to be seen that day, depending on the availability of counselors/staff. However, recently the CWC has implemented a new system while switching to the telehealth appointment system. In this new system, students can call the CWC and arrange an initial consultation with a counselor shortly after calling. Thus, with the new system, students are guaranteed to be seen for their first non-emergency appointment within 24 hours, at a time that works best for them. This new system hopes to increase both the timeliness of first non-emergency appointments and the number of first appointments the CWC can accommodate. Emergency appointments by phone or walk-in are addressed within minutes of arrival/calling.

It is important to note that both the switch to a telehealth appointments system and general effects from COVID-19 may have impacted appointment demand at the CWC. This research analyzes and compares appointment demand data before the shift to telehealth (i.e., the Fall semesters from 2015 through 2019) with appointment demand data after the shift to telehealth (i.e., Fall semester 2020) to determine if there was any significant change in appointment demand. The research only compares Fall semesters because the only entire semester of telehealth appointment demand data available at the time of the analysis was Fall 2020. Changes in appointment demand include both changes in the overall amount of demand (i.e., is there a higher demand for appointments in a telehealth system?) and changes in demand pattern (i.e., is there a shift in the weekday or time of day when appointment demand is highest?).

Previous research on this topic falls under two categories: previous research on demand for telehealth appointments due to COVID-19, and previous research relating to appointment demand at UF's CWC. Previous research relating to demand for telehealth appointments during the COVID-19 pandemic has found an overall increase in the use of telehealth appointments. The Center for Disease Control's Morbidity and Mortality Weekly Report reported a “ $154 \%$ increase in telehealth visits during the last week of March 2020, compared with the same period in 2019" (Koonin et al., 2020, p. 1598). This increase may be, in part, due to COVID-19 socialdistancing recommendations (Koonin et al., 2020).

Previous research relating to appointment demand at UF's CWC was concerned with predicting future appointment demand using a machine learning algorithm (Magee et al., 2019). This research distinguishes itself from this previous research and builds off of it. This research 
uses telehealth appointment demand data and analyzes changes in demand instead of predicting future demand. Furthermore, this research categorizes appointments as specific types and analyzes the demand for each appointment type. However, this research does build off the previous appointment demand research done with the CWC, mainly in how some of the data was categorized before analysis. These details are further discussed and cited in the data analysis section.

In general, the team hypothesized there would be an overall increase in demand for appointments when looking at the telehealth appointment data compared to the historical nontelehealth appointment data. The team used two main points to formalize this hypothesis. First, the telehealth system allows students to call the CWC and schedule a first appointment at a time that is most convenient for them. The team predicted this would lead to an increase in demand for appointments. Next, the telehealth system was implemented at the same time as the COVID19 pandemic. The team predicted that the potential for increased isolation during the COVID-19 pandemic (ex: social distancing policies) could lead to an increase in demand for mental health service appointments.

This research hopes to help the CWC understand how their demand may have changed from switching to a telehealth appointment system due to COVID-19. By better understanding their demand, the CWC can better equip themselves to handle first appointments efficiently and timely. The results of this research can help ensure that the UF population members receive the mental health support they need as quickly as possible.

\section{Data Analysis}

Both historical non-telehealth appointment demand data and telehealth appointment demand data was analyzed from a 6-year period. For both the historical non-telehealth data and the telehealth data, appointments were separated by time of the semester and then analyzed based on appointment type, day of the week, and time of day.

\section{Time of Semester}

The raw data was first separated based on the time of the semester. Previous research found that the first two weeks of Fall classes tend to have higher demand than the rest of the Fall semester (Magee et al., 2019). Thus, each semester was split up into two major categories: the 
first two weeks of classes, and the rest of the semester, not including reading days and finals week.

To account for some years having a holiday during the first two weeks of classes, the first two weeks of a semester is considered the first 10 days of classes. There are two exceptions due to missing data: the first two weeks of Fall classes for 2016 and the first two weeks of Fall classes for 2019. In both cases, there was appointment data for only 9 of the first 10 days of classes.

When analyzing historical non-telehealth appointment data, the first two weeks of classes for the Fall semesters from 2015-2019 were analyzed together, and the rest of the Fall semesters from 2015-2019 were analyzed together, meaning that the raw data was grouped together based on time of the semester and analyzed as a whole.

\section{Categorizing Appointment Types}

After the raw data was separated based on the time of semester, the appointments were grouped based on appointment type. There are four main appointment types a given appointment can fall under: triage, consultation, phone/email, and paperwork alert. This can also be seen in Figure 1. It is important to distinguish between different appointment types since they each take a different amount of time, on average, to complete. Also, by categorizing the appointments by type, the team can better analyze demand patterns for specific appointment types.

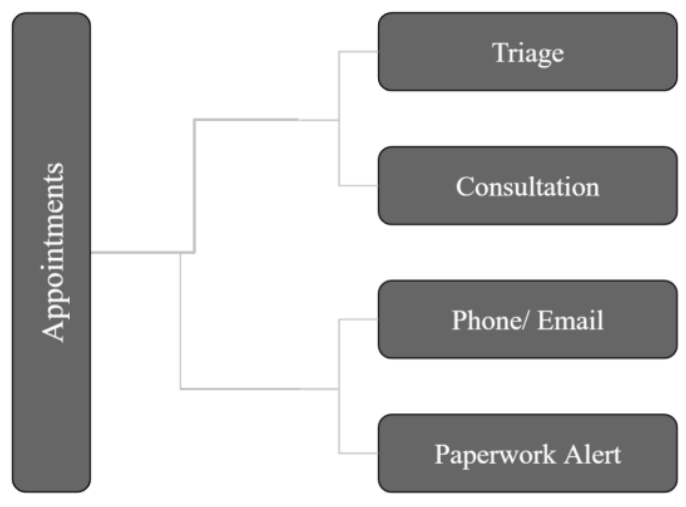

Figure 1. Diagram of Appointment Types

For each appointment, the raw data included a brief description indicating if the appointment was a consultation, triage etc. For example, an appointment description could say "On-Call Non- 
Client Consultation" or "On-Call Client Consultation". Although both appointments fall under the general category of consultations, they have different descriptions in the raw data. Having different descriptions for appointments of the same type makes it difficult to find trends for each appointment type. Thus, to group appointments that fall under the same appointment type, each appointment was categorized as one of the four appointment types mentioned earlier. Categorizing appointments was done primarily using VBA code in excel. The code looped through all the raw data appointments and categorized the appointment as a triage, consultation, phone/email, or paperwork alert based on the keywords in the appointment description. This allowed all triages, all consultations, all phone/email, and all paperwork alerts appointments to be analyzed together even if their respective appointment descriptions varied slightly.

\section{Time of Day and Weekday}

The data was analyzed based on the time of day and the weekday. It is important to note that the main results do not include any outlier appointments. Outlier appointments are any appointments before 8 am Monday through Friday, or any appointments 5pm or later Monday through Friday. Outlier appointments also include any appointments on the weekends, regardless of time. The results do not include these appointments because these appointments are not representative of typical appointments offered by the CWC and may skew the demand data. The data filtered out outlier appointments mainly using pivot tables in excel.

The team analyzed the average number of appointments for each appointment type for each weekday, and for each working hour between 8 am-5 pm. The following equations were used: Average Number of Appointments Per Weekday j for Appointment Type $i=\frac{\sum_{t=1}^{t=9} P_{i, j, t}}{D_{j}}$ Average Number of Appointments Per Hour $t$ for Appointment Type $i=\frac{\sum_{j=1}^{j=5} P_{i, j, t}}{\sum_{j=1}^{j=5} D_{j}}$

$$
\begin{gathered}
P_{i, j, t}=\text { Number of appointment type } i, \text { on weekday } j, \text { at time } t \\
\qquad \begin{array}{c}
D_{j}=\text { Total number of } \text { weekday } j \text { in data set } \\
t=1(8 \text { AM }), \ldots, 9(4 \text { PM }) \\
j=1(\text { Monday }), \ldots, 5 \text { (Friday) } \\
i=1 \text { (Triage }), \ldots, 4 \text { (Paperwork Alert) }
\end{array}
\end{gathered}
$$


The team also wanted to compare results from each appointment type with the results for all the appointment types together. For example, comparing the average number of Triage appointments on Mondays with the average number of all appointment types on Mondays (triage, consultation, phone/email, and paperwork alert). When looking at the average number of appointments per weekday and per hour for all appointment types, the following equations were used:

Average Number of Appointments for Weekday $j$ for All Appointments $=\frac{\sum_{t=1}^{t=9} P_{j, t}}{D_{j}}$

$$
\begin{gathered}
\text { Average Number of Appointments for Hour } t \text { for All Appointments }=\frac{\sum_{j=1}^{j=5} P_{j, t}}{\sum_{J=1}^{j=5} D j} \\
P_{j, t}=\text { Number of appointments on weekday } j \text { at time } t
\end{gathered}
$$

It is important to note that the results do not examine the average number of appointments per weekday for the first two weeks of classes. This is because when looking at the data through the lens of the weekday, there was no significant change or general pattern in the demand based on weekday for the first two weeks. Instead, the results breakdown the daily average number of appointments for each appointment type. To calculate the daily average number of appointments for each appointment type, the following equation was used:

$$
\begin{gathered}
\text { Daily Average Number of Appointment Type } i=\frac{\sum_{t=1}^{t=9} R_{i, t}}{\sum_{j=1}^{j=5} D_{j}} \\
R_{i, t}=\text { Number of appointment type } i \text {, at time } t
\end{gathered}
$$

\section{Results}

\section{First Two Weeks of The Fall Semester}

The first two weeks of the Fall semester saw both a shift in appointment demand pattern and a slight change in the overall demand for appointments. When comparing demand throughout the day between the historical non-telehealth appointment data and the telehealth appointment data, the team found there was a shift in demand. In the historical non-telehealth appointment data, there was a slight preference for appointments in the morning, with a peak of an average of 7 appointments at $11 \mathrm{am}$, as seen in Figure 2. As opposed to the telehealth appointment demand, 
where there was a higher demand in the afternoon, with a peak of an average of 10 appointments at $1 \mathrm{pm}$, as seen in Figure 3. It is important to note that both of these peaks consider all appointment types (i.e. triage, consultation, phone/email, and paperwork alert).

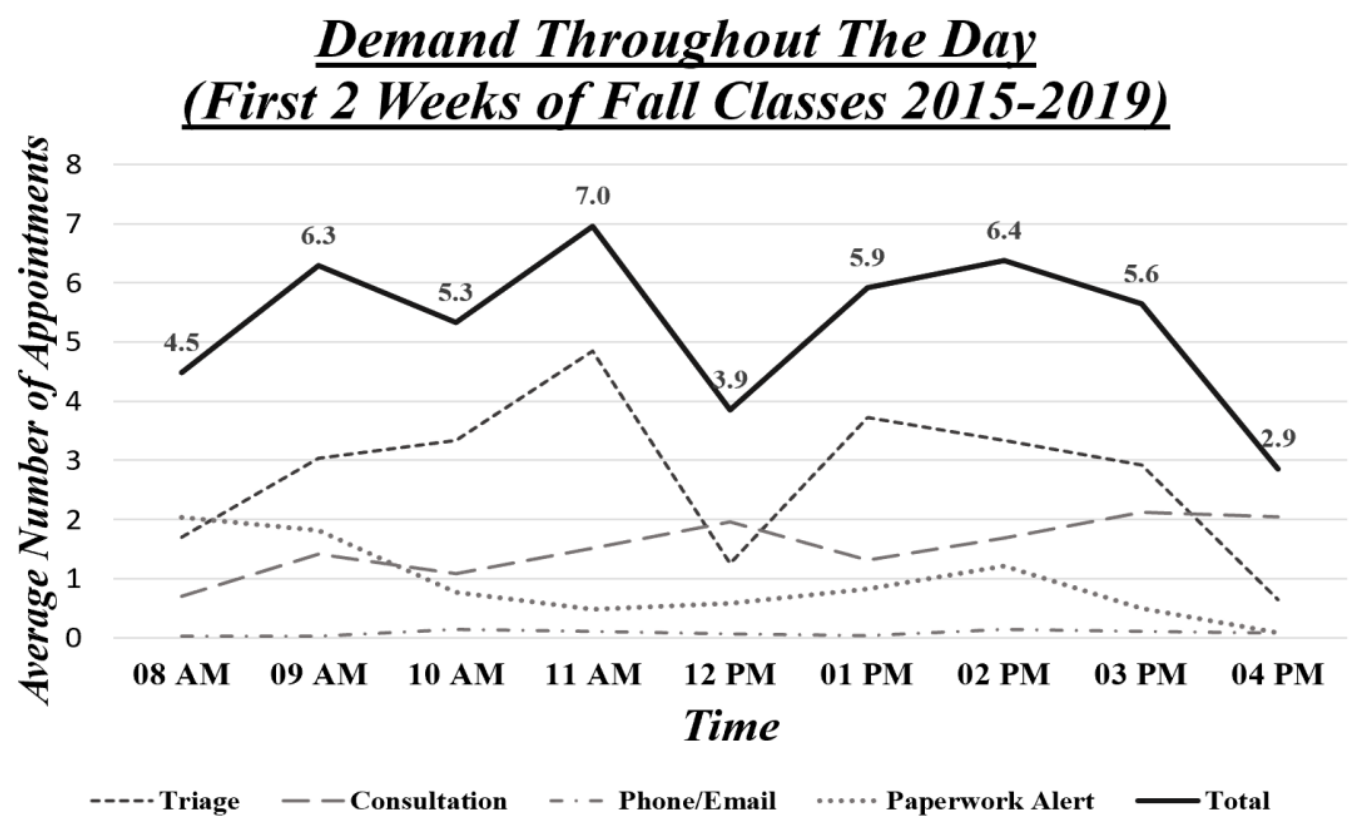

Figure 2. Average Appointment Demand Per Hour in Historical Non-Telehealth Data, First Two Weeks of Fall Classes 


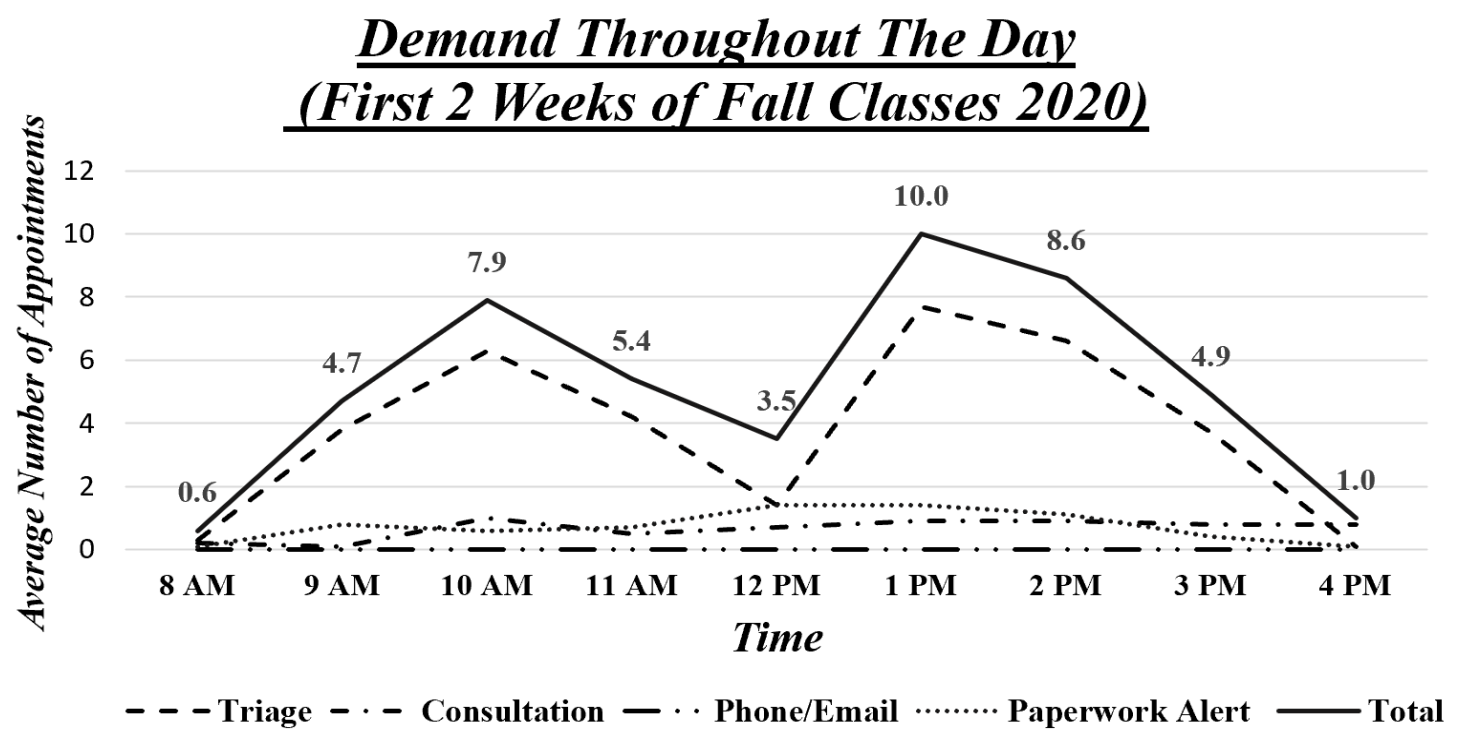

Figure 3. Average Appointment Demand Per Hour in Telehealth Data, First Two Weeks of Fall Classes

Next, comparing the breakdown of appointment types, the team found a shift here as well. In the new telehealth appointment system, there is a major increase in the number of triages. In the telehealth appointment system $73 \%$ of the daily average number of appointments were triages, as opposed to $52 \%$ in the non-telehealth appointment system. In the telehealth appointment system, there was also a significant decrease in phone/email appointments. In fact, when looking at the overall daily average, there were no phone/email appointments in the telehealth system. The full breakdown of the daily average number of appointments for each appointment type can be seen in Figure 4 for historical non-telehealth data and in Figure 5 for the telehealth data. It is important to note that this change in the daily average number of appointments for each appointment type may be due to the CWC's new system implemented during the shift to telehealth, and may not be due to an actual change in demand. 
Daily Average Appointment Type Breakdown

(First 2 Weeks of Fall Classes 2015-2019)

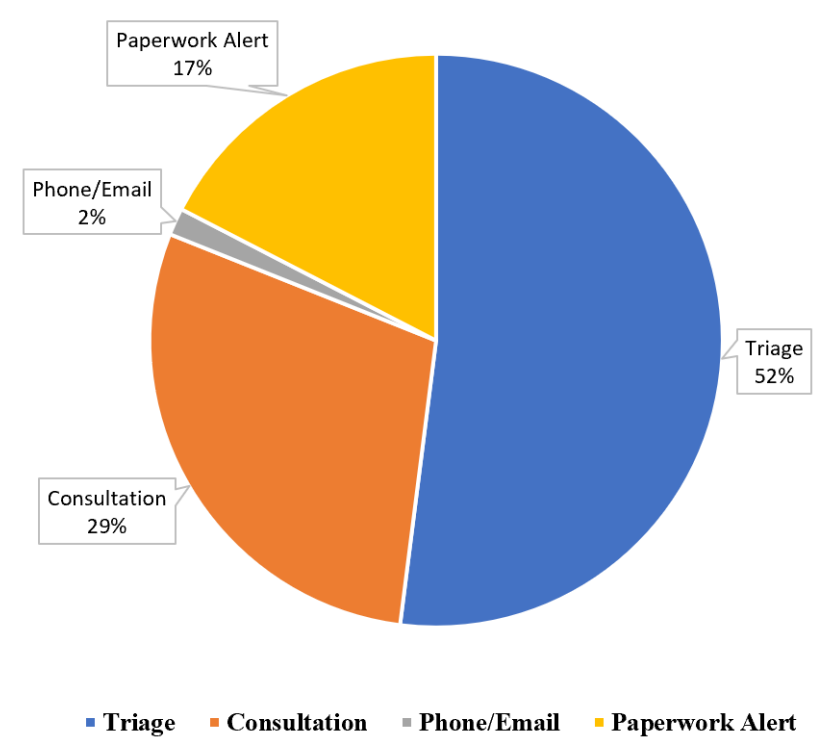

Figure 4. Daily Average Appointment Type Breakdown for Historical Non-Telehealth Data, First Two Weeks of Fall Classes

\section{Daily Average Appointment Type Breakdown}

(First 2 Weeks of Fall Classes 2020)

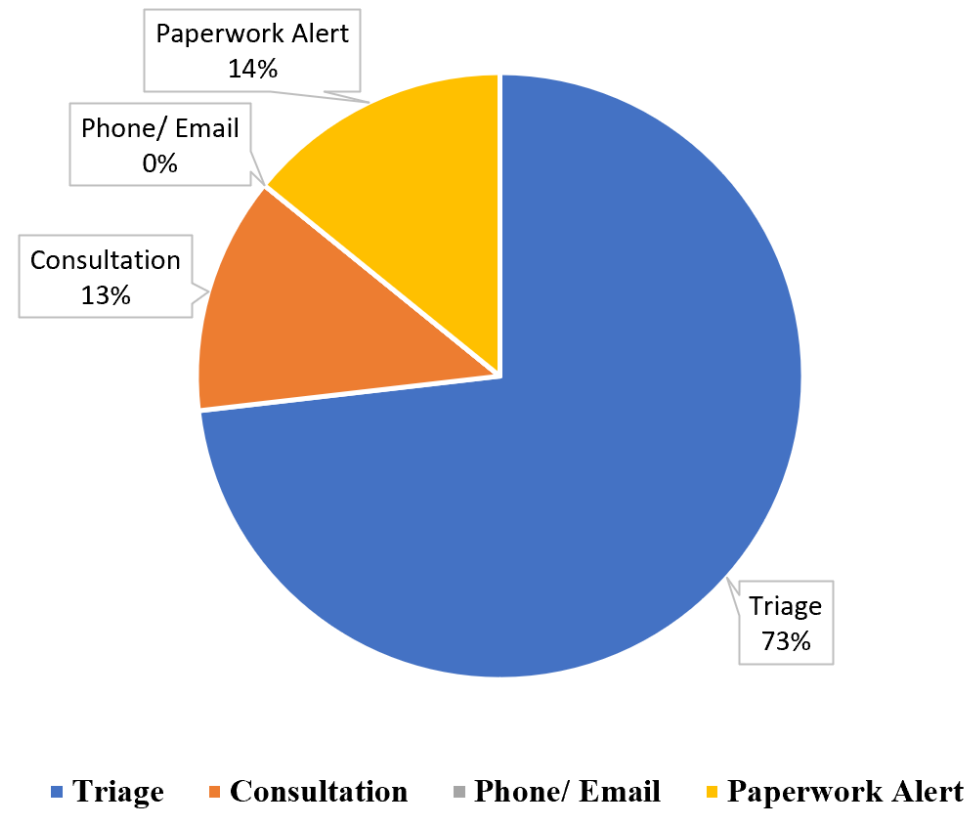

Figure 5. Daily Average Appointment Type Breakdown for Telehealth Data, First Two Weeks of Fall Classes 
Lastly, there was a slight decrease in the total number of appointments when comparing the historical non-telehealth appointment data and the telehealth appointment data for the first two weeks of the Fall semester. In the historical non-telehealth appointment data, there was a daily average number of appointments of 48 , whereas the telehealth appointment data had a daily average number of appointments of 47 . This results in a $2 \%$ decrease in the daily average number of appointments when looking at all appointment types together. The team conducted a two-sample t-test assuming unequal variances. The p-value was 0.712 for a two-tail test with an alpha of 0.05 . Therefore, the daily average number of appointments between the non-telehealth and the telehealth appointment data was not significant.

\section{Rest of The Fall Semester}

When looking at all appointment types together for the rest of the Fall semester, there was a slight shift in demand patterns between the historical non-telehealth appointment data and the telehealth appointment data. In the historical non-telehealth appointments, there was a higher demand for appointments in the morning, with a peak of an average of 6.7 appointments at 11 am, as seen in Figure 6. In the telehealth appointment demand, there is a slightly higher demand in the afternoon for appointments, with a peak of an average of 6.1 appointments at $2 \mathrm{pm}$, as seen in Figure 7.

\section{Demand Throughout The Dav} (Fall Classes Excluding the First 2 Weeks 2015-2019)

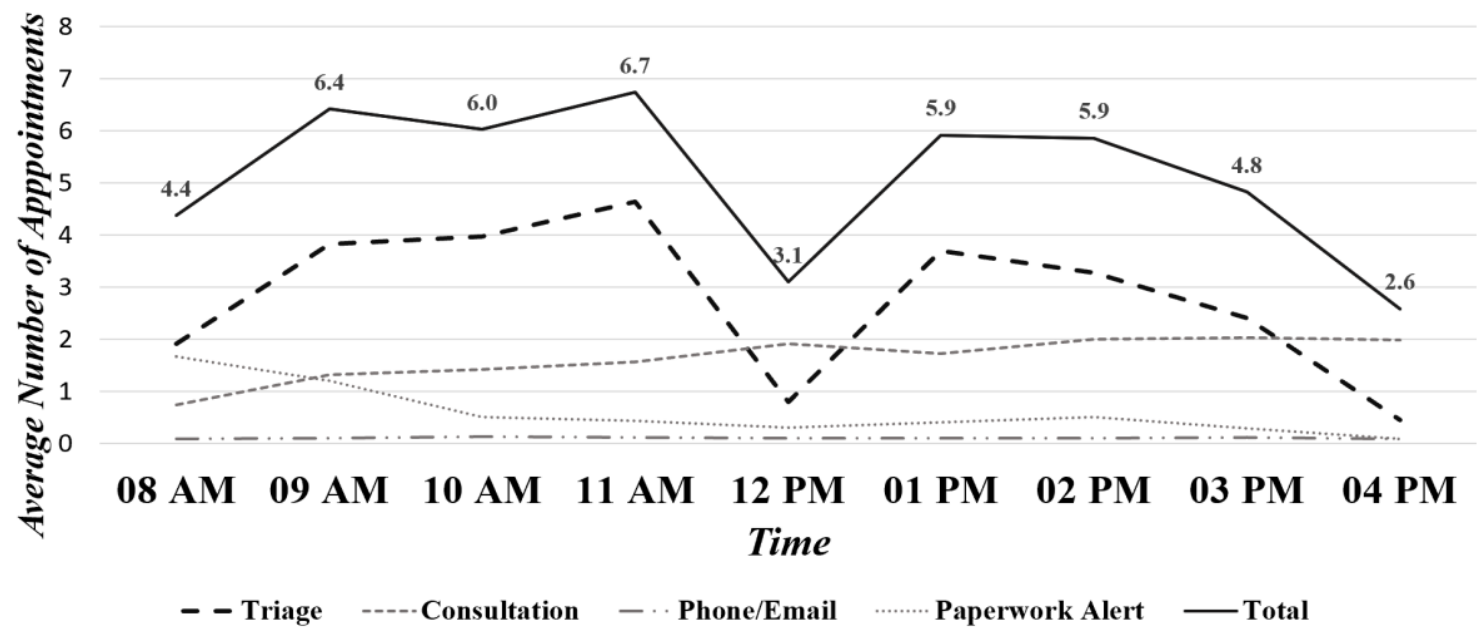

Figure 6. Average Appointment Demand Per Hour in Historical Non-Telehealth Data, Rest of Fall Semester 


\section{Demand Throughout The Day}

\section{(Fall Classes Excluding the First 2 Weeks 2020)}

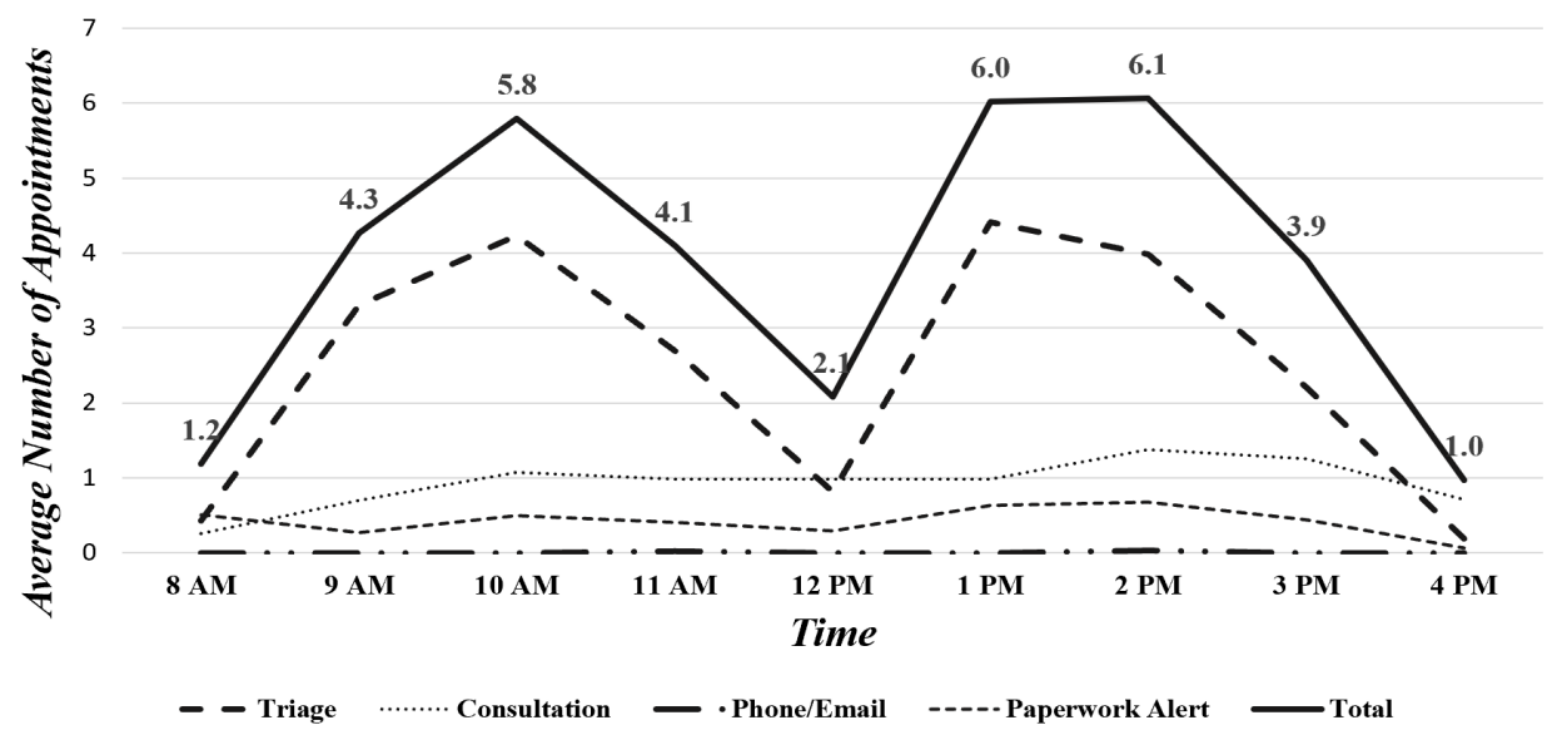

Figure 7. Average Appointment Demand Per Hour in Telehealth Data, Rest of Fall Semester

Furthermore, there was a shift in demand patterns throughout the week. In the historical nontelehealth appointment demand, Monday has the highest demand based on the average number of appointments, and demand slowly decreases with Friday having the least demand, as seen in Figure 8. This is when looking at all appointment types together. In the telehealth appointment data, Monday still has the highest demand, but demand no longer continuously decreases with Friday being the least busy day. Demand dips with the least busy day being Wednesday, and then continues to increase slightly both Thursday and Friday. This trend can be seen in Figure 9, and again is considering the average number of all appointment types together. 


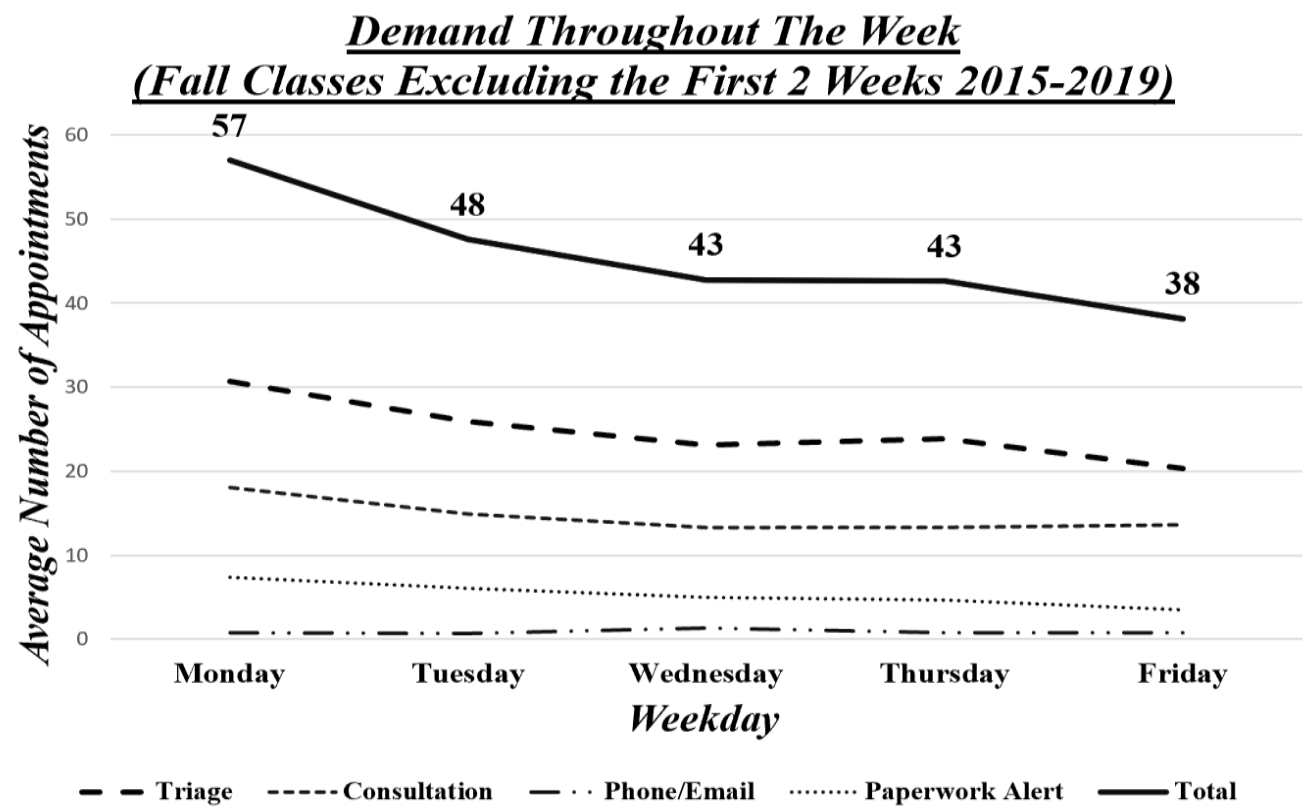

Figure 8. Average Appointment Demand Throughout the Week, Historical Non-Telehealth Data, Rest of Fall Semester

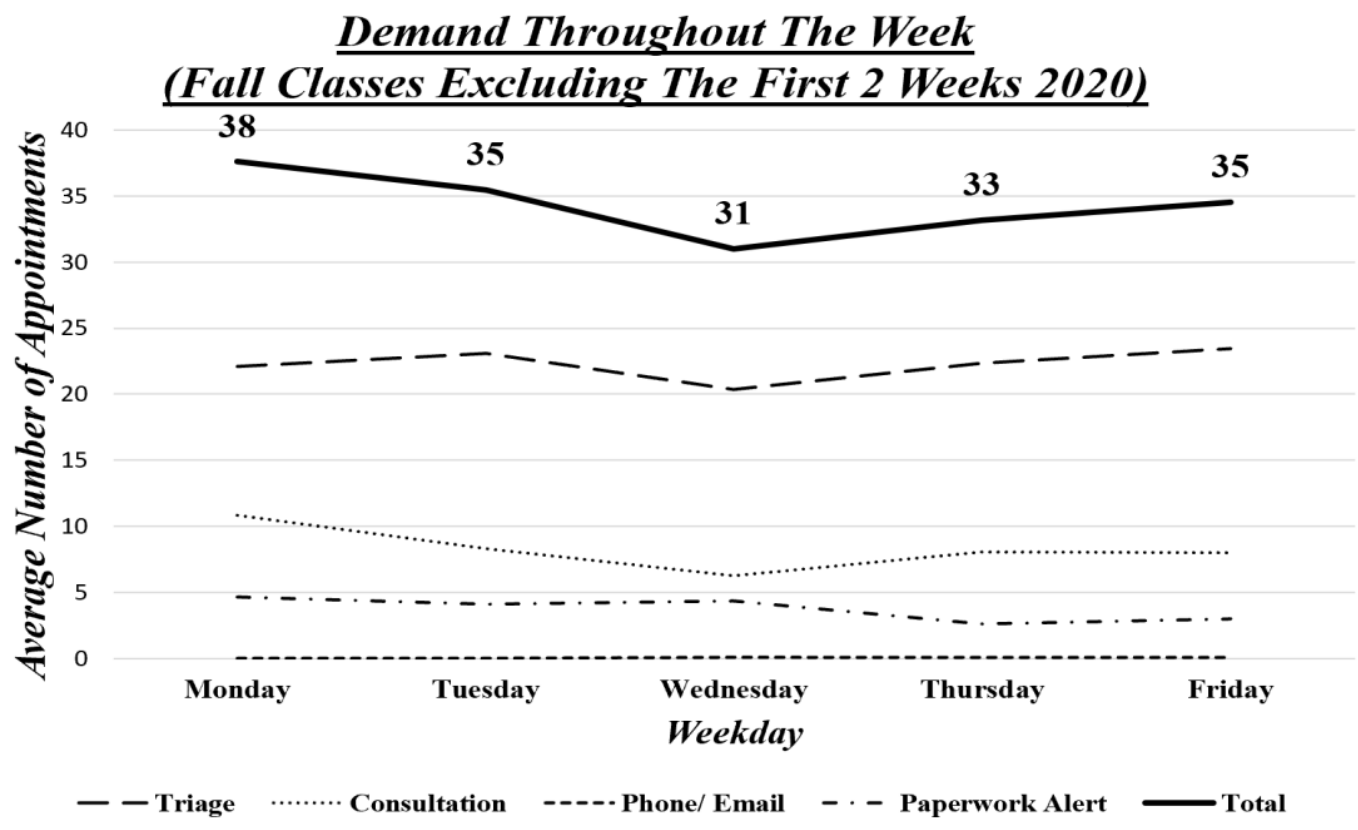

Figure 9. Average Appointment Demand Throughout the Week, Telehealth Data, Rest of Fall Semester

The historical non-telehealth and telehealth appointment data also showed a change in daily average appointment type breakdown. The main change was an increase in the percentage of 
daily average appointments that were triages, from 54\% in the historical non-telehealth data, to $65 \%$ in the telehealth appointment system. A full comparison of daily average appointment type breakdown can be seen in Figure 10 for historical non-telehealth data, and Figure 11 for the telehealth appointment data. Again, it is important to note that this shift in daily average appointment type breakdown may be due to the new system the CWC has implemented, as opposed to a change in demand.

Daily Average Appointment Type Breakdown (Fall Classes Excluding First 2 Weeks 2015-2019)

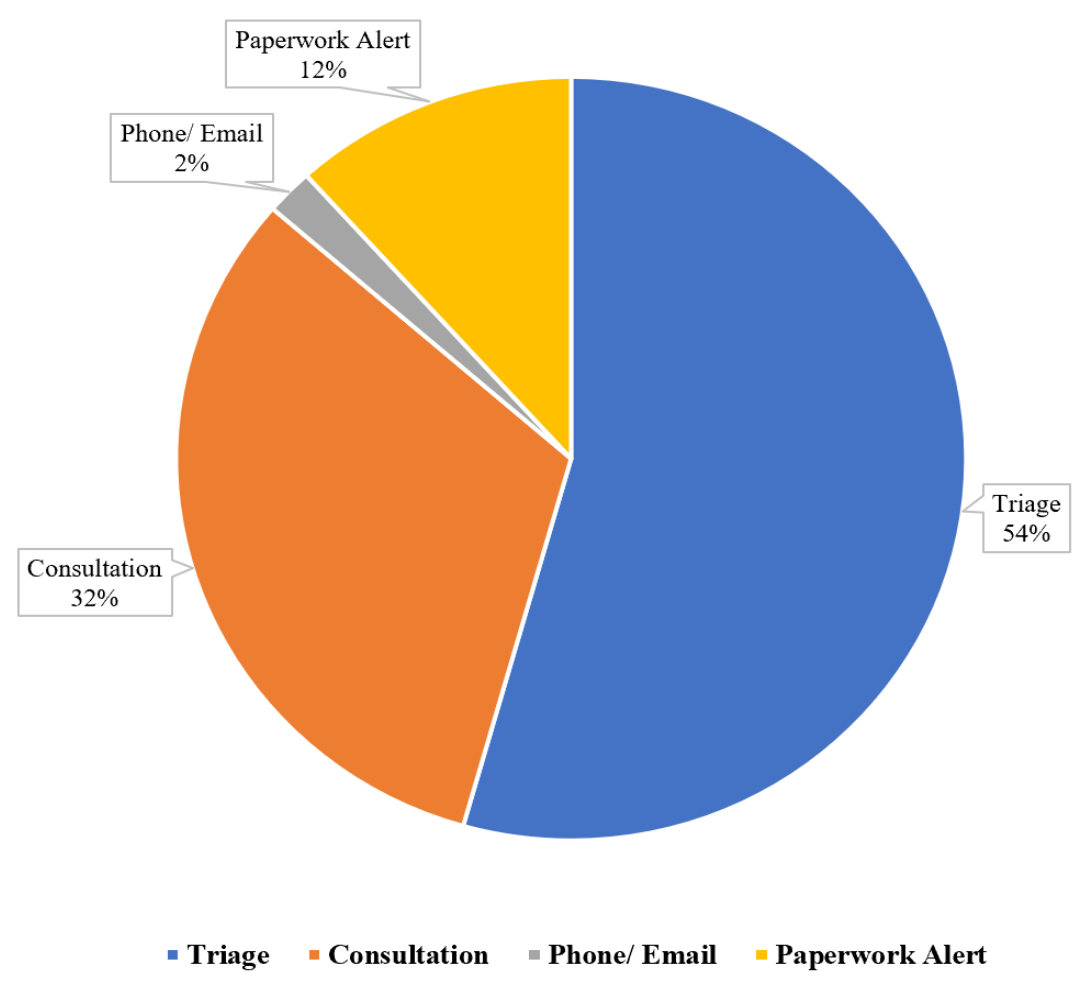

Figure 10. Daily Average Appointment Type Breakdown for Historical Non-Telehealth Data, Rest of Fall Semester 


\section{Daily Average Appointment Type Breakdown} (Fall Classes Excluding First 2 Weeks 2020)

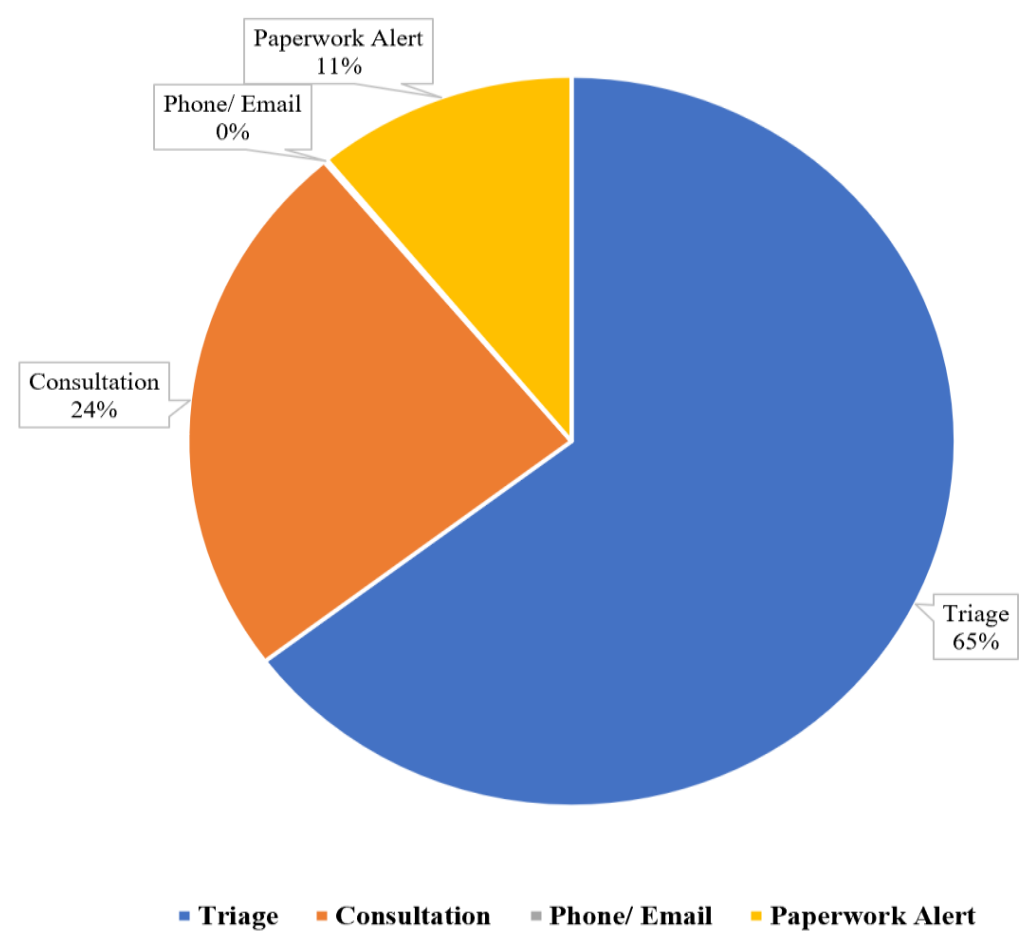

Figure 11. Daily Average Appointment Type Breakdown for Telehealth Data, Rest of Fall Semester

Box and whisker plots were created to examine the overall spread of daily appointment demand for each day of the week. The box and whisker plots show the minimum number of daily appointments, the maximum number of daily appointments, and the $25^{\text {th }}, 50^{\text {th }}$, and $75^{\text {th }}$ percentiles of the daily number of appointments. There is one box and whisker plot for each day of the week. Figure 12 shows box and whisker plots for historical non-telehealth data. Figure 13 shows box and whisker plots for telehealth data. Outliers for a given weekday appear as dots above or below the respective box and whisker plot. When looking at the historical nontelehealth appointment demand, one can see that the daily number of appointments is more spread out for each day of the week than the telehealth appointment demand. Overall, the team found that appointment demand in the telehealth appointment system was smoother than that of the non-telehealth appointment system. 


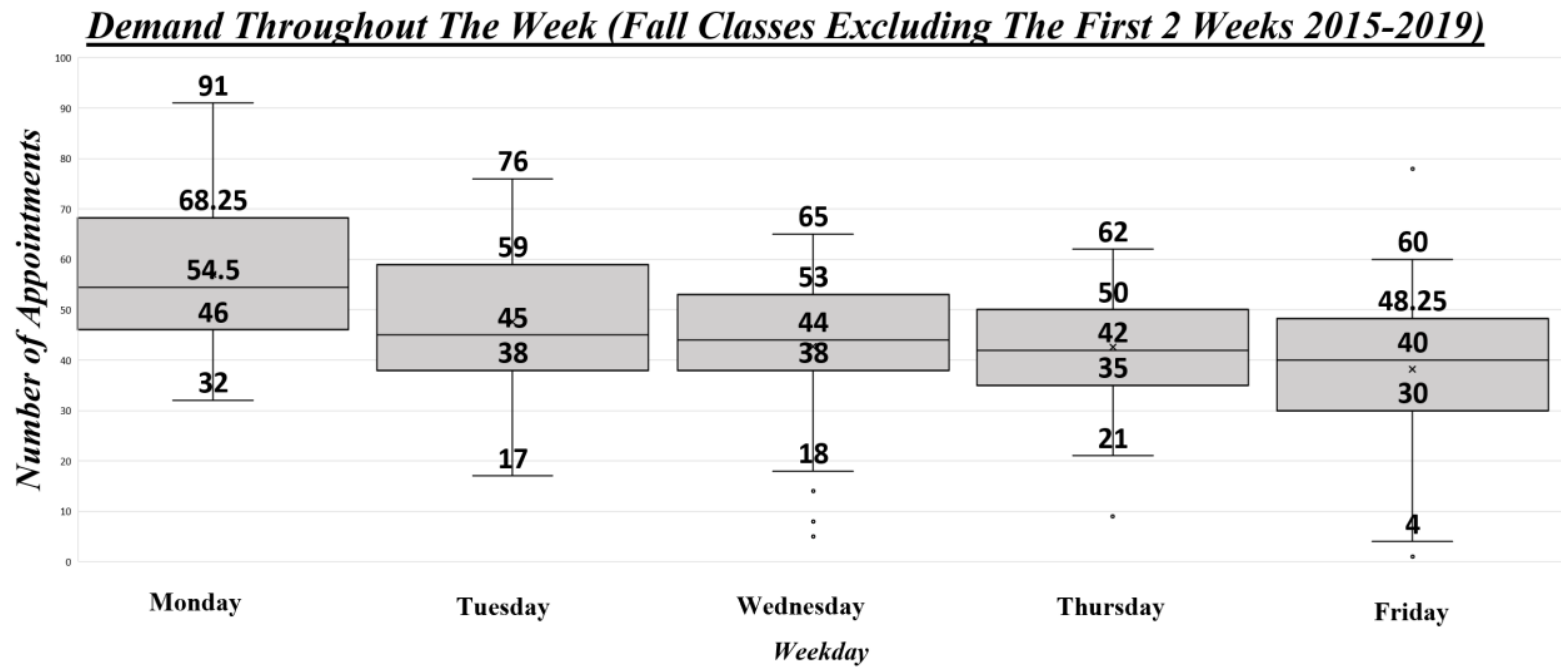

Figure 12. Spread of Appointment Demand for Each Day of the Week, Historical Non-Telehealth Data

Demand Throughout The Week (Fall Classes Excluding The First 2 Weeks 2020)

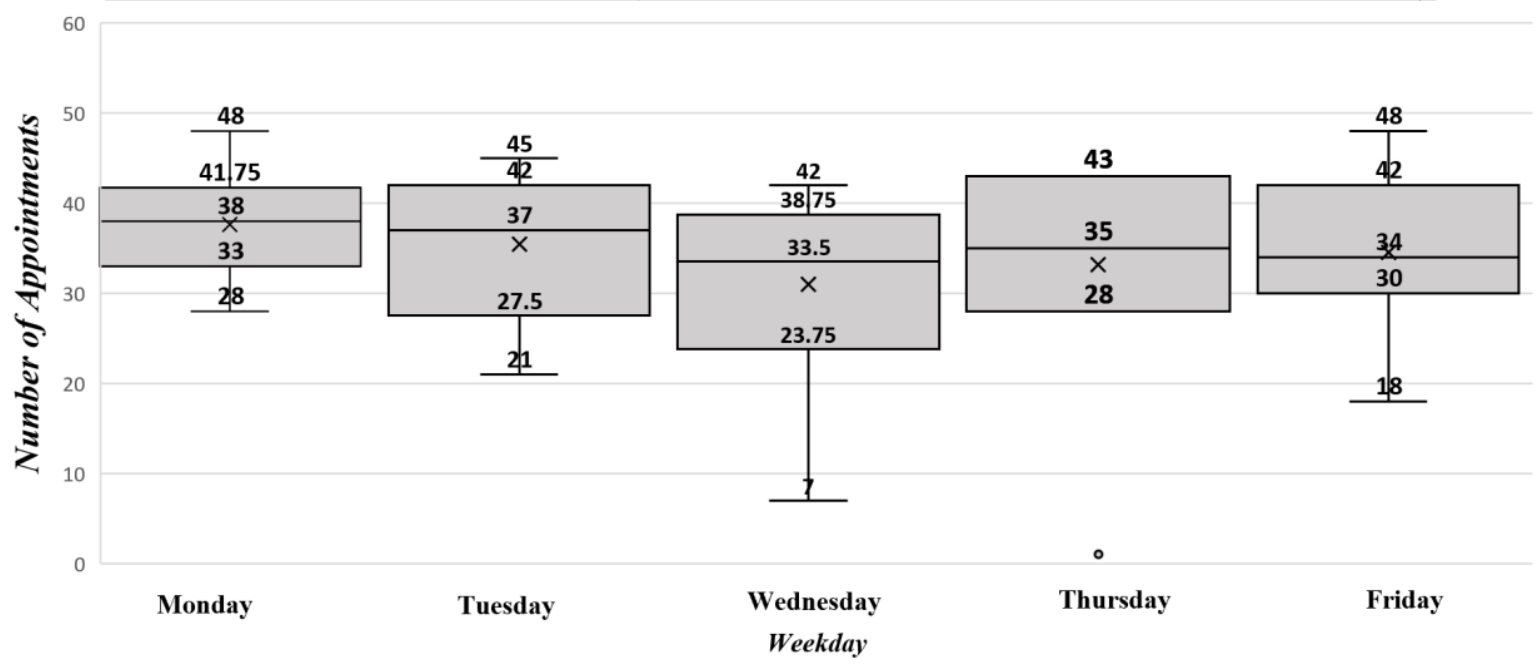

Figure 13. Spread of Appointment Demand for Each Day of the Week, Telehealth Data

Lastly, there was a significant decrease in appointment demand in the telehealth appointment system for the rest of the Fall semester. When looking at the historical non-telehealth appointment system, there was a daily average of 46 appointments. There was a daily average of 34 appointments in the telehealth appointment system, which a $26 \%$ decrease in appointment demand. A two-sample t-test with unequal variances was conducted. The t-test results had a p- 
value of $1.155 \mathrm{E}-12$ for a two-tail test with an alpha of 0.05 . Therefore, the daily average number of appointments between the non-telehealth and the telehealth data was significant for the Fall semester.

\section{Discussion and Conclusion}

When comparing the telehealth appointment data to the non-telehealth appointment data, the team's initial hypothesis that there would be an increase in the total demand for appointments in the telehealth system was wrong. When looking at the telehealth appointment demand data, there is a slight decrease of $2 \%$ in the daily average number of appointments during the first two weeks and a more significant decrease of $26 \%$ in the daily average number of appointments during the rest of the Fall semester. Furthermore, there is a general shift in demand pattern, where telehealth appointments had higher demand in the afternoon, as opposed to historical nontelehealth appointments that tended to have higher demand in the mornings.

These changes in demand can be due to many factors. First, the shift to higher demand in the afternoon may be due to the shift of classes from in-person to Zoom, due to the pandemic. Since most students have their classes over Zoom, they may not be waking up as early to go to classes since most students do not need to account for any commuting time to campus. Since students may not be waking up as early as they previously did, this may have increased demand for appointments later in the day.

Furthermore, there is an overall decrease in demand for appointments in the telehealth system. This too can be caused by many factors. One factor may be that since the telehealth system is new at the CWC, it will take time for word to spread about this new service throughout the UF student population.

The team hopes this research will provide the CWC with insight as to how their appointment demand has changed from to the shift to a telehealth system due to the COVID-19 pandemic. As a result, the CWC may be better equipped to handle their appointment demand in a timely manner, and more members of the UF population can receive the support they need. 


\section{References}

Koonin, L. M., Hoots, B., Tsang, C. A., Leroy, Z., Farris, K., Jolly, B., ... \& Harris, A. M. (2020). Trends in the use of telehealth during the emergence of the COVID-19 pandemic - United States, JanuaryMarch 2020. Morbidity and Mortality Weekly Report, 69(43), 1595.

Magee, E., Karaca, M., Alvarado, M., Escoto, E., \& Lawrence, A. (2019). Machine Learning Applied to Walk-in Demand Prediction of a University Counseling Center. UF Journal of Undergraduate Research, 2l(1).

United States Department of Health \& Human Services. What is telehealth? (Updated 2021). Retrieved March 24, 2021, from https://telehealth.hhs.gov/patients/understanding-telehealth/\#what-istelehealth 